Service social

\title{
La décentralisation après la loi de 1991 sur les services de santé et les services sociaux au Québec
}

\section{Luciano Bozzini et Jacques Bourgault}

Volume 41, numéro 2, 1992

Bilan des réformes

URI : https://id.erudit.org/iderudit/706571ar

DOI : https://doi.org/10.7202/706571ar

Aller au sommaire du numéro

Éditeur(s)

École de service social de l'Université Laval

ISSN

1708-1734 (numérique)

Découvrir la revue

Citer cet article

Bozzini, L. \& Bourgault, J. (1992). La décentralisation après la loi de 1991 sur les services de santé et les services sociaux au Québec. Service social, 41(2), 87-114. https://doi.org/10.7202/706571ar
Résumé de l'article

Après six ans de consultations diverses, le chapitre 42 des lois du Québec de 1991 présente sous l'appellation de décentralisation une réponse aux demandes d'autonomie budgétaire et programmatique des régions et des établissements. Cet article analyse, à partir du texte de loi et des critères reconnus, dans quelle mesure il s'agit d'une réelle décentralisation ; il revoit en outre le chemin parcouru à cet égard depuis le rapport de la Commission Castonguay-Nepveu et met en relief les enjeux de pouvoir et d'autonomie liés à la décentralisation dans les rapports entre trois catégories d'acteurs : le MSSS, les régies régionales et les établissements. Enfin, l'article relève trois « tests de vérité » que la réforme subira sous peu et qui révéleront la portée réelle du transfert de pouvoirs vers les régions : la réglementation de la loi, les premières règles budgétaires et leur application, de même que les transferts d'effectif du MSSS vers les régions. 


\section{La décentralisation après la loi de 1991 sur les services de santé et les services sociaux au Québec}

Luciano BOZZINI

Professeur, Département d'administration de la santé

Université de Montréal

Jacques BOURGAULT Professeur, Département de science politique Université du Québec à Montréal Professeur associé à I'ENAP

\section{INTRODUCTION}

Depuis l'adoption de la Loi sur les services de santé et les services sociaux et modifiant diverses dispositions législatives (projet de loi 120 devenu le chapitre 42 des Lois du Québec de 1991), la tant discutée et attendue réforme du secteur sociosanitaire est enfin mise en application. Au fil des mois, depuis le rapport Rochon déposé au printemps de 1988, plusieurs documents gouvernementaux et avant-projets de loi sont venus alimenter le débat sur la réforme qui s'enclenche. Toutes les principales pièces préalables au projet de loi adopté - rapport Rochon, Orientations Lavoie-Roux (Québec, 1989), 
document Côté (Québec, 1990) - ont proposé, malgré des différences d'un document à l'autre, une option de base : le renforcement du système public. À l'encontre des appels répétés à plus de privatisation provenant de divers idéologues et groupes d'intérêts, le gouvernement a décidé en faveur du maintien d'un système sociosanitaire essentiellement public qui, au vu des données, a relativement bien servi les intérêts de la population québécoise'.

Il est également utile de rappeler que ce choix de base a fait l'objet d'un consensus généralisé2 .

Le renforcement visé de la décentralisation est un élément clef de la réforme. Depuis la parution du rapport Rochon, du reste, le plaidoyer en faveur d'une plus grande décentralisation régionale a été une constante de tous les documents gouvernementaux. En cela, la leçon de la Commission aurait été retenue : la centralisation excessive a en effet été la plainte la plus souvent formulée par presque tous les acteurs au moment des consultations de la Commission. Centralisation excessive entraînant tout un cortège d'effets pervers : bureaucratisation (lenteurs, inadaptation des décisions, déresponsabilisation et démotivation des professionnels et des citoyens), court-circuitages du palier régional, absence d'intégration locale. Dans la même veine, le réseau sociosanitaire et l'État lui-même ont subi les effets pervers du mode " corporatif » et centralisé d'allocation des fonds publics : chaque groupe a essayé de maximiser ses gains propres, dans des confrontations plus ou moins transparentes avec l'État. Le rapport Rochon concluait avec pertinence : "le Ministère ne peut gérer 900 établissements depuis Québec » et " Le système est en otage aux groupes d'intérêts ».

Le débat centralisation-décentralisation de la prise de décision dans les systèmes sociosanitaires publics n'est pas propre au Québec. D'une part, divers pays occidentaux ont été en mesure de constater les effets pervers d'une trop grande centralisation de la prise de décision (Field, 1989 ; Saltman et von Otter, 1992). D'autre part, dans le cadre d'un système public ayant des objectifs nationaux d'équité (financement, normes de services, etc.), certaines décisions reviennent par définition au centre.

Par ailleurs, la réponse au couple centralisation-décentralisation est contrainte par l'organisation politico-administrative et par les traditions historiques de chacun des pays. À titre d'exemple, les pays scandinaves ont une longue tradition de financement/management régional et local dans le secteur sociosanitaire, ce qui n'est pas le cas dans d'autres pays. Autre exemple : des variations importantes sont observables entre les provinces canadiennes ; en Alberta, les municipalités ont conservé un rôle important dans les affaires sociosanitaires, ce qui n'est pas le cas au Québec.

Au Québec, déjà la Commission Castonguay-Nepveu avait plaidé pour une régionalisation conséquente. L'histoire des vingt dernières années montre toutefois que pour plusieurs raisons qui seront analysées plus loin, la décen- 
tralisation n'a pas été aussi loin que l'on aurait souhaité. En même temps, la conviction de la nécessité d'une plus grande décentralisation est aujourd'hui plus forte que jamais et le gouvernement a annoncé dans ses documents récents de politiques (Lavoie-Roux, 1989 : 107 et ss. ; Côté, 1990 : 47 et ss.) son intention de décentraliser.

D'où les deux questions centrales qui seront traitées dans le présent texte. La loi de 1991 va-t-elle entraîner une réelle décentralisation de la prise de décision vers le niveau régional ? Et quels sont les éléments contextuels qui contraignent le développement de la décentralisation?

La première section de ce texte propose une grille $d^{\prime}$ analyse permettant de juger du degré de décentralisation inhérent à une organisation politicoadministrative donnée. La deuxième section retracera les grandes lignes de l'évolution de la décentralisation dans le secteur sociosanitaire, de la réforme Castonguay au rapport de la Commission Rochon. La troisième section sera consacrée à une analyse des récentes propositions de décentralisation et de la loi de 1991 en tant qu'instrument juridico-administratif de la réforme. Finalement, les perspectives de la décentralisation dans le nouveau contexte seront évaluées.

\section{LA NATURE DE LA DÉCENTRALISATION : GRILLE D'ANALYSE}

L'organisation des rapports entre le lieu de décision stratégique et le point de prestation des services publics a grandement nourri la controverse ; d'une part, les modèles centralisés prévoient la prise de décision autant que la prestation des services à partir d'un seul et même point central ; c'est le cas par exemple d'organismes centraux tels le Conseil du trésor et le ministère des Finances. À l'autre extrême du continuum existent des modèles organisationnels décentralisés qui prévoient que la prise des grandes décisions se fait dans I'instance même qui livre les services ; entre ces deux pôles, on trouve des formes d'organisation centralisées, mais relativement déconcentrées, dotées de deux niveaux d'instances, l'une, centrale, prenant les décisions stratégiques de financement, de programmation et d'organisation et l'autre dans les points de services décidant des modalités opérationnelles et exécutant, cas par cas, les politiques décidées centralement. Dans le cas des services sociaux et de santé québécois, les systèmes décentralisés et déconcentrés ont tenté les concepteurs des réformes.

La littérature montre l'importance des enjeux du débat, en particulier ceux de l'autonomie et de l'imputabilité à la population régionale (Gélinas, 1975 : 4-21). Dans le cas de la décentralisation territoriale complète, l'organe régional trouve son financement et établit ses budgets en conséquence ; il décide de ses objectifs, de sa programmation, de son organisation, de son 
recrutement. II n'en est redevable qu'à la population régionale qu'il dessert. Dans le cas de la déconcentration territoriale, l'organe régional a plutôt un rôle d'exécutant local des politiques nationales, avec des objectifs et une programmation établis par le centre ; dans ce cas, l'organisation, les procédés de travail et le recrutement sont établis dans chacun des bureaux régionaux en fonction des politiques du centre, lesquelles politiques peuvent laisser plus ou moins de marge de manœuvre aux instances régionales qui sont redevables pour l'exécution à l'instance centrale et non pas aux populations locales.

La décentralisation et la déconcentration peuvent être territoriales ou techniques. Dans le premier cas, la souveraineté nationale dans un domaine est divisée en espaces régionaux qui jouissent d'une structure déconcentrée ou décentralisée ; il y a décentralisation technique lorsque la souveraineté en ce domaine est déléguée par voie législative à un organe spécialisé qui jouit de cette autonomie, dans un secteur précis, mais sur tout le territoire. C'est le cas des sociétés d'État. Il y a déconcentration technique lorsqu'un organisme central ou déjà décentralisé divise sa compétence en domaines de spécialisation technique : les directions générales sont des formes de déconcentration technique.

Quatre critères ${ }^{3}$ permettent de distinguer les formes décentralisées et les formes déconcentrées d'organisation : I'existence d'une personnalité juridique propre, la détention d'un champ de compétence exclusive, l'autofinancement et la sélection des dirigeants par la population desservie ou par I'organisme lui-même. Plus un organisme satisfera à ces quatre critères et plus il le fera complètement, plus on pourra le qualifier de décentralisé. Dans le cas contraire, on le considérera comme déconcentré.

De fait, compte tenu à la fois du cadre juridique et des transactions administratives quotidiennes non exemptes de relations de pouvoir avec les autres organismes de l'environnement organisationnel, peu d'organismes satisfont pleinement à ces quatre critères et peu ne satisfont aucunement à aucun de ces critères. Il existe donc un continuum dont les deux pôles sont la centralisation parfaite et la décentralisation totale de la tutelle de l'organisme central, chaque instance s'y situant selon la façon dont elle « vit » les quatre conditions. Par exemple, si les commissions scolaires semblent satisfaire aux quatre critères, il ne faut pas oublier à quel point les subventions normées et les agrégations de programmes et d'établissements limitent leur marge de manœuvre ; à l'inverse, si les bureaux régionaux des ministères ne semblent satisfaire aucun des critères, on doit savoir que la tutelle de la capitale sur les bureaux régionaux varie en intensité d'un ministère à l'autre.

Il apparaît que l'exercice de qualification doit se faire en deux étapes : d'une part, la qualification par rapport à l'organe de souveraineté nationale ferait du ministère de la Santé et des Services sociaux une déconcentration technique du gouvernement en raison de la collégialité de l'autorité du 
Conseil des ministres, et une commission scolaire serait une décentralisation territoriale. Dans une seconde étape, on peut qualifier l'organe de prestation du service : ainsi le bureau régional de Trois-Rivières d'Hydro-Québec serait une déconcentration territoriale d'une décentralisation technique, tandis que la direction générale de l'informatique de la commission scolaire serait une déconcentration technique $d^{\prime}$ une décentralisation territoriale. Une telle entreprise de qualification à deux paliers a l'avantage de distinguer deux niveaux d'enjeux de pouvoir et d'éviter la confusion classique survenant lorsqu'on veut qualifier simultanément la prestation des services et la prise de décision programmatique.

Le premier critère de distinction porte sur l'existence juridique de I'organisme à qualifier : I'organisme décentralisé doit exister juridiquement du fait de la création par une loi ou de l'incorporation en vertu d'une loi générale existante (Dussault et Borgeat, 1984 : 57) ; le bien-fondé de ce critère apparaît d'évidence. Comment l'organe qui n'existe pas pourrait-il avoir des pouvoirs ? Ainsi l'ambassade ou le bureau régional d'un ministère n'existent pas séparément de la personnalité juridique de l'organisme dont ils dépendent, le pays ou le ministère.

L'exercice d'un champ de compétence exclusive qui confère une souveraineté matérielle à l'organisme détenteur se trouve décrit dans la loi habilitante ou la charte de l'organisme ; c'est à l'intérieur de ce champ de compétence que l'organisme exerce sa souveraineté, mais il peut se trouver que d'autres lois plus générales, comme celles créant les organismes centraux ou régissant des paliers administratifs (Loi sur les cités et villes), fixent des bornes ou des conditions à l'exercice de ce champ de compétence. Ainsi, des bureaux régionaux de ministères n'auront pas la compétence de décider le contenu des politiques et des programmes, bien que dans plusieurs cas ils puissent être chargés de leur exécution. Le cas des sociétés d'État est particulier à cet égard puisque, depuis les années 80, les organismes centraux tels le ministère du Conseil exécutif et le Conseil du trésor appliquent une politique de mandats auprès des organismes et des personnes qui siègent aux conseils d'administration ; les organismes centraux veulent ainsi, au nom du pouvoir de direction, de coordination et de contrôle du gouvernement, forcer la main des sociétés d'État quant aux programmes à implanter, aux politiques de tarifs et de coûts et à la détermination de la marge bénéficiaire à retourner à l'actionnaire, le gouvernement.

L'adage qui veut que l'argent soit le nerf de la guerre trouve pleinement son application dans le troisième critère. L'autofinancement de l'organisme lui confère une autonomie de programmation et de gestion. Cet autofinancement peut venir de la capacité d'imposer et de taxer (municipalités et commissions scolaires) ou du pouvoir de gérer ses recettes (sociétés d'État) et de déterminer la disposition de ses surplus (sociétés d'État du Québec d'avant 
1980). Lorsque I'organisme fonctionne à même un budget déterminé centralement et versé selon le calendrier et les conditions de l'instance centrale, sa marge de manœuvre s'en trouve réduite, de même que sa capacité décisionnelle ; il s'agit alors d'un cas de déconcentration. Dans les cas de décentralisation, il faut déterminer quelle est la part du financement qui est «autonome » et quelles sont les conditions que des instances centrales (les ministères ou le Conseil du trésor) imposent aux organismes pour la préparation, la dépense et le contrôle budgétaire ; dans les cas où le financement extérieur à l'organisme « décentralisé » se révèle important du fait de subventions conditionnelles (normées), il convient de qualifier de « relative » I'autonomie financière de l'organisme décentralisé et, conséquemment, son indépendance.

La sélection des dirigeants constitue l'ultime critère de qualification de la décentralisation : on convient en effet que les dirigeants doivent leur loyauté à qui les choisit, les nomme et peut régir leur carrière. Ainsi, les processus d'élection des dirigeants municipaux et de commisisons scolaires satisfont au critère de décentralisation, alors que la dotation centrale des postes de directeurs régionaux ne le fait pas. La situation des sociétés d'État est plus complexe : les présidents et membres du conseil d'administration sont nommés pour un mandat limité dans le temps par le gouvernement, quelquefois même sur la proposition du ministre ; ils doivent donc leur affectation à une instance centrale. En revanche, les postes de cadres dans ces sociétés sont en général dotés selon les politiques corporatives et non pas selon les règles centrales de la Loi sur la fonction publique ; ils doivent donc allégeance à l'organisme lui-même. En somme, une fois la nomination gouvernementale effectuée à la très haute direction, l'organisme est en principe maître de son processus de dotation... sauf si des organismes centraux ont le pouvoir d'imposer des normes et conditions ou encore si le ministre de tutelle intervient à cet effet ou enfin si les mandataires du gouvernement au conseil d'administration respectent un mandat que le gouvernement leur a confié à cet effet.

\section{Avantages et inconvénients des modèles}

La décentralisation territoriale qui permet à la population d'un territoire d'exercer un contrôle politique de type démocratique sur la direction de l'organisme présente le principal avantage de laisser les citoyens concernés prendre, par l'intermédiaire de leurs représentants élus, les décisions qui leur semblent opportunes en matière de programmation et de tarification directe (coût d'utilisation) ou indirecte (taxation). Le contrôle politique sur la distribution des services devient ainsi plus direct, immédiat et régulier ; le bien public qui est produit a plus de chances d'être adapté aux besoins de la population que l'administration connaît mieux du fait du meilleur flux de communications entre la population, les dirigeants politiques et les fonctionnaires. Le service peut donc être plus efficace. 
La population desservie perçoit qu'elle a un meilleur accès aux dirigeants politiques et aux fonctionnaires de ces plus petites unités administratives, ce qui l'encourage à réagir plus souvent et plus rapidement qu'elle ne le fait à l'égard des grandes administrations centrales. Ce processus permet donc une plus grande participation des citoyens à la gouverne de la société et, par conséquent, une meilleure intégration politique des citoyens et une plus grande responsabilisation des contribuables-consommateurs.

Le processus de décision décentralisé augmente la transparence dans la production des biens publics, puisque le citoyen sait mieux qui a eu l'initiative des projets, qui a fait pression, qui a participé à la décision, quels étaient les alternatives et leurs coûts. En outre, il permet une meilleure imputabilité des dirigeants politiques et administratifs face à une population mieux informée et disposant, outre les élections, de réels moyens politiques de contrôle, comme c'est le cas par exemple des assemblées des conseils municipaux et des comités d'arrondissements.

Les plus petites unités administratives qui caractérisent les organismes décentralisés sont dotées de processus de gestion taillés sur mesure en fonction du bien produit, de la taille de l'organisme et des valeurs, traditions et culture de la population desservie. Elles bénéficient aussi de plus de souplesse de gestion et de plus de rapidité de réaction pour s'adapter aux besoins qui changent ou aux modifications dans l'environnement de la distribution des services. L'efficacité du service et son efficience risquent ainsi de s'accroître (Aucoin et Bakvis, $1988:$ :111).

L'émulation régionale est aussi un effet bénéfique de la décentralisation : chaque unité poursuit librement son effort de recherche et crée de meilleures formules... que les autres imiteront au vu de leur succès ; tandis que la concentration force la spécialisation des tâches et induit le monopole des agents centraux sur des domaines de réflexion.

Les modèles relativement centralisés qui comportent des formes de déconcentration territoriale du pouvoir présentent aussi de nombreux avantages dont le principal est l'équité nationale dans la fourniture de biens publics. En effet, lorsqu'un programme est conçu pour l'ensemble de la population d'un État et financé par l'ensemble de sa population, on n'observe pas les effets pervers dus à la capacité financière de chaque région de se doter du service puisque son financement opère une forme de péréquation nationale ; par ailleurs, les normes nationales du programme servent de standards pour garantir partout sur le territoire la fiabilité de la disponibilité et de la qualité des services.

Ces modèles permettent aussi de réaliser des économies d'échelle dans I'approvisionnement en matières premières, dans l'encadrement de la production des biens publics et en raison de l'unicité de l'instance de décision. La concentration des ressources permet aussi de former des masses critiques 
porteuses d'effets synergiques : une concentration de chercheurs peut s'avérer plus stimulante et productive que la dispersion de ces mêmes chercheurs en unités isolées sur le territoire. De plus, la gestion centralisée réduit dans certains cas les coûts d'interconnexion et de coordination entre les entités régionales et favorise la complémentarité des initiatives : quelle serait l'utilité pour un organisme régional de santé de combattre une épidémie que ses voisins refuseraient de reconnaître?

Les modèles centralisés permettent également l'existence de normes de gestion et de contrôles nationaux qui diminuent les cas de corruption et d'irrégularités administratives. Ils associent finalement la prestation du bien public à l'instance centrale de décision, renforçant l'identité nationale, le sentiment d'appartenance et donc l'intégration et la cohésion de l'État national.

Bref, toute médaille a son revers et il n'est pas de situations qui soient exclusivement porteuses d'avantages. Pour choisir entre les modèles centralisés ou décentralisés, on doit se laisser guider par les valeurs politiques recherchées, la spécificité du bien public à fournir et l'importance à accorder à la participation locale des usagers, des contribuables, des gestionnaires et des prestateurs. Par ailleurs, en vertu d'une loi d'airain de la vie sociale, il faut bien voir que chaque groupe d'acteurs concerné (citoyens, groupes de pression, fonctionnaires, professionnels, gestionnaires) aura tendance à proposer une formule et à soutenir une dynamique favorisant ou à tout le moins protégeant ses intérêts propres.

\section{L'EXPÉRIENCE DE LA DÉCENTRALISATION, DU RAPPORT CASTONGUAY AU RAPPORT ROCHON}

On ne peut saisir la portée et les chances de la loi de 1991 en ce qui concerne la décentralisation sans un regard sur le passé et l'expérience de la dernière décennie.

Un des traits majeurs de la réforme proposée par la Commission Castonguay-Nepveu consistait dans la transformation d'un système sociosanitaire pluraliste, décentralisé et géré/contrôlé par des organismes privés (même si le financement était largement public) en un système à toutes fins utiles "nationalisé », un réseau intégré avec une gouverne centrale forte.

En contrepartie, la Commission tenait un discours soutenu sur la nécessité d'une forte décentralisation régionale pour les mêmes raisons qu'on invoque aujourd'hui : adaptabilité, efficience, nécessité de la coordination / intégration régionale et locale, responsabilisation des acteurs régionaux et locaux, motivation des professionnels, participation de la population à la fois au développement social et à la gestion des services sociosanitaires. En ligne avec ce discours, la Commission Castonguay (Québec, 1970) préconi- 
sait la création d'Offices régionaux de la santé (ORS) et d'Offices régionaux des services sociaux (ORSS) détenant des mandats et des pouvoirs importants : planification, programmation, allocation des crédits d'exploitation aux établissements et organismes, approbation des immobilisations, création, fermeture et fusion d'établissements, pouvoirs de réglementation. Significativement, les ORS et les ORSS sont présentés comme des « agents de la collectivité régionale ». Bref, au départ, à la fois l'idée de gouverne et de contrôle centraux et de décentralisation de certaines fonctions en vue d'une meilleure efficience, adaptation, intégration étaient présentes ${ }^{4}$.

On sait qu'en ce qui concerne la décentralisation, la loi 65 de 1971, même pilotée par M. Castonguay, resta très en deçà des propositions de la Commission. Dans sa version finale, un organisme mixte pour la santé et les services sociaux vit le jour - les Conseils régionaux de la santé et des services sociaux (CRSS) - organisme dont les principaux mandats furent ainsi définis : susciter la participation de la population ; assurer des communications entre le public, le ministre et les établissements ; conseiller les établissements ; faire des recommandations au ministre ; promouvoir les échanges de services, les services communs et l'intégration des services sur le territoire. Bref, on passait des ORS/ORSS dotés de pouvoirs importants à des CRSSS presque uniquement consultatifs ${ }^{5}$.

En commission parlementaire, $\mathrm{M}$. Castonguay fut toutefois entièrement explicite sur cette mise en déroute du projet original de la Commission sur la décentralisation : pour expliquer la timidité de son projet de loi, il invoqua I'absence de compétences administratives suffisantes, l'inexistence d'un palier politico-administratif régional doté de pouvoirs de taxation et garant d'imputabilité et la nécessité, dans un premier temps, de contrôler centralement la rationalisation du système et l'évolution des coûts (Turgeon, $1983: 46)$.

\section{L'expérience des CRSSS}

Au fil des années - faisant suite à l'augmentation des compétences en région, au développement d'outils de planification et de gestion, à une certaine " crédibilité » des CRSSS - le Ministère augmentera progressivement les mandats confiés aux conseils régionaux, la plupart de ceux-ci allant toutefois dans le sens de leur intégration dans l'appareil administratif du Ministère. On notera pour mémoire ici : I'approbation des critères d'admission et de sortie des bénéficiaires; le suivi et le contrôle des mesures de désengorgement des urgences ; la stimulation de la mise en commun de services et d'achats de groupe, etc. Des mandats plus conséquents du point de vue de l'autonomie de gestion ou, à tout le moins, de la capacité d'influence régionale ont toutefois été confiés aux CRSSS dans les années plus récentes. Les CRSSS 
ont été chargés de la préparation des « Plans régionaux de services »(PROS) pour la santé mentale et la réadaptation. Leur rôle dans I'allocation des ressources est progressivement accru : ils peuvent être appelés à co-gérer - en concertation avec les établissements et le Ministère - I'allocation des crédits de développement dans les divers programmes. Par ailleurs, la mise sur pied en 1977 des " commissions administratives régionales » pour les différents établissements ou programmes - lieu de rencontre entre le conseil régional et les établissements et organismes régionaux - a constitué un tournant important dans la consolidation des CRSSS. Il n'existe pas d'évaluation systématique de cette expérience, mais selon plusieurs observateurs (entre autres, Bhérer et al., 1986), cette stratégie a permis le renforcement du CRSSS comme un intermédiaire "forcé » entre les établissements et le Ministère. Plusieurs CRSSS en ont tiré parti pour s'établir comme " interlocuteurs valables » à la fois auprès des établissements et du Ministère.

Malgré ce renforcement progressif des CRSSS, la décentralisation de la prise de décision a toutefois été très insuffisante entrainant, sur le plan régional, les effets pervers classiques déjà énumérés. Par ailleurs, la centralisation administrative excessive a eu un effet de paralysie sur les fonctions prioritaires auxquelles le Ministère, selon de nombreux témoignages à la Commission Rochon, devrait se consacrer. Largement occupé à gérer 900 établissements depuis Québec, le Ministère a été contraint de négliger ses fonctions stratégiques propres, celles ayant, à moyen et à long terme, le plus d'impact sur le système : la coopération et la planification intersectorielle, la définition des politiques et moyens fondamentaux du système, l'innovation, la péréquation régionale, la planification/formation de la main-d'œuvre et la coopération inter-professionnelle, la répartition des effectifs sur le territoire, l'évalution et le contrôle des résultats, etc. On pourrait également faire une longue liste des problèmes qui perdurent depuis des années et que le Ministère n'a pas su ou n'a pas trouvé le temps de résoudre : la pénurie de lits pour malades chroniques, l'engorgement des urgences, I'intégration locale insuffisante des services, la dégradation des conditions de travail dans les établissements qui affecte le moral des employés comme des gestionnaires, etc.

Il est nécessaire ici de discuter brièvement de ce qui a probablement été le facteur décisif qui a empêché une extension plus significative de la décentralisation. L'argument de M. Castonguay sur l'absence d'un niveau politicoadministratif régional a en fait lourdement pesé sur le développement des conseils régionaux tout au long des vingt dernières années. Cette absence de répondant politique institutionnalisé au niveau régional, avec pouvoir de taxation - qui aurait pu permettre une décentralisation, partielle au moins, de I'imputabilité - a empêché que soit élaboré un équilibre satisfaisant entre un contrôle politique et administratif central nécessaire et une part d'autonomie régionale souhaitable. En conséquence, le centre a été avare dans la délégation d'autorité et de pouvoirs aux conseils, cela produisant une sorte de cercle 
vicieux : les établissements (en particulier les plus puissants) ont joué avec la faiblesse du palier régional en le court-circuitant, par le canal administratif (en recourant directement au Ministère), ou par le canal politique (par l'intermédiaire des politiciens locaux). Cela a eu pour effet d'affaiblir le palier régional, le processus renforçant la tendance du centre à ne pas déléguer. Au-delà de la centralisation indue des responsabilités quant aux décisions de planification et de programmation régionales, le centre est même tombé dans le piège de la centralisation des responsabilités et décisions opérationnelles. Bref, on a assisté à une centralisation à la fois publique, corporatiste et administrative des enjeux et problèmes locaux.

Cette impossibilité d'instaurer un débat politique et administratif local a été exacerbée par les caractéristiques des membres des conseils d'administration des conseils régionaux. Jusqu'en 1981, ces derniers étaient largement composés de représentants des établissements, ce qui faisait des conseils une arène surtout occupée au partage du gâteau, au lieu d'être des forums de décision branchés sur les besoins des citoyens (donc également sur les intérêts des députés locaux) grâce à une présence significative des citoyens aux conseils d'administration. Avec le chapitre 27 (1981), la situation s'est améliorée un peu (les membres hors réseau comptant alors pour environ la moitié), mais il n'existe pas de données fermes sur les effets de ces changements. Plusieurs observateurs avancent la thèse d'une influence encore prépondérante du réseau sur les décisions (à travers également la présence des citoyens nommés par le ministre, I'influence des commissions administratives d'établissements et celle des permanents du réseau).

Au bilan, il est globalement vrai que les CRSSS n'ont pas été investis de I'autorité correspondant aux deux décisions stratégiques clés : la définition des orientations et objectifs du système et l'autorité de définir et de contraindre les moyens (allocation des budgets et ressources) pour l'atteinte des objectifs. Si globalement il s'impose de constater plus une déconcentration $q u$ une décentralisation de l'action, cela ne signifie pas, d'une part, que les conseils ont été impuissants et incapables de remplir un rôle utile et, d'autre part, que les douze conseils régionaux se sont développés conformément à un seul prototype. Dans les mots de la Commission :

...Tout le monde s'accorde pour dire que le processus décisionnel à l'intérieur du système est trop centralisé et que le système est trop " gros »... Le système devient tellement complexe, réglementé, embourbé, traversé par des conflits qu'il est à toutes fins pratiques ingouvernable...

Un nouveau partage du pouvoir doit être établi entre le centre et les régions... Cette nécessité repose autant sur le constat des faiblesses du passé que sur la perception de faits porteurs d'avenir et sur les défis inévitables qui doivent être relevés à moins de vouloir s'enliser dans le statu quo. Malgré les insatisfactions qui s'expriment à l'égard 
des CRSSS, il reste qu'ils ont contribué à ce que s'affirment à I'intérieur du système les réalités régionales : désormais, toute planification doit tenir compte des spécificités des régions et de leur apport respectif. De plus, il existe un mouvement de concertation à l'intérieur des régions et des sous-régions qui constitue un acquis, une force et une promesse pour l'avenir. Enfin, on peut affirmer que les établissements se reconnaissent dans les mécanismes de planification et de décision mis en œuvre par les CRSSS. Du côté du Ministère, la prise de conscience de son engorgement amène une revalorisation de la planification et une accentuation des efforts nécessaires à la mise en oeuvre de mécanismes d'évaluation efficaces et utiles... (Québec, 1988 : 495-496)

De plus, comme une des rares études existant sur la performance des CRSSS semble l'indiquer (Bhérer et al., 1986), l'absence de modèle rigide de développement des CRSSS ou ce qu'on pourrait nommer la « phase expérimentale » dans le développement des conseils a permis l'accumulation de leçons utiles, mais également l'émergence de modèles différenciés de conseils régionaux. II semblerait que trois types-idéaux ont émergé de l'expérience des vingt dernières années. Certains conseils se rapprocheraient du modèle du bureau déconcentré du Ministère (ou modèle top-down). L'enracinement de ces conseils (auprès des établissements et de la population) est limité ; ce genre de conseil est « faible » et candidat typique au courtcircuitage par les producteurs ou les groupes de citoyens. Un deuxième groupe se rapprocherait plutôt du modèle de la " fédération d'établissements » (ou modèle bottom-up). Ces conseils jouent la carte de porte-parole des organisations auprès du centre en travaillant sur des consensus régionaux et en négociant avec les établissements des demandes « raisonnables » adressées au centre. Un troisième groupe de conseils se rapprocherait du modèle du « gouvernement régional ». Ces conseils travaillent également sur le mode bottom-up en ajoutant cependant à la représentation des producteurs celle des intérêts de la population. Ces conseils maximiseraient en quelque sorte leur légitimité locale (et leur pouvoir réel par opposition à leur autorité légale). Une autre recherche (Bégin et al., 1987) témoigne de l'écart classique entre I'aspect formaliste des choses (p. ex. des structures défavorables à la décentralisation) et la réalité : dans certaines régions, les capacités de leadership du conseil régional comme les dynamismes locaux ont permis à la décentralisation d'aller plus loin que dans d'autres, où les conseils étaient pourtant dotés de la même autorité légale !

En fait, ces succès relatifs et cette consolidation progressive des conseils régionaux - à noter également que de 1978 à 1988, alors que les budgets des différentes catégories d'organismes du réseau sociosanitaire augmentaient en moyenne de $122 \%$, celui des CRSSS a augmenté de 317 \% (Bélanger, 1990) - représentent I'assise pratique de la nouvelle politique de décentralisation mise de l'avant dans la réforme qui s'enclenche. 


\section{DE LA COMMISSION ROCHON À L'ADOPTION DE LA LOI DE 1991 : DISCOURS ET RÉALITÉ DE LA DÉCENTRALISATION}

La formule adoptée à la suite de la réforme Castonguay était une sorte de " compromis technocratique et législatif » fondé sur la réalité et les perceptions de l'époque : absence d'identités régionales fortes et de compétences administratives en quantité suffisante, absence d'un niveau politico-administratif régional imputable. Deux changements en particulier rendent plus mûre aujourd'hui l'idée d'un accroissement de l'autonomie régionale. D'une part, comme l'a souligné la Commission Rochon, depuis 1970 les dynamismes régionaux - économiques, politiques, identitaires - se sont intensifiés. Même si la nature et la direction du développement régional au Québec et des politiques gouvernementales à cet égard sont loin d'être définies, on assiste, dans certaines régions au moins, à un renforcement du tissu social, des compétences, du sentiment d'appartenance, de la volonté d'auto-développement régional et local ${ }^{6}$.

En tout état de cause, la Commission Rochon a reçu un message clair sur l'émergence de "conscience et volonté régionales » dans le secteur sociosanitaire. Par ailleurs, la prise de conscience des lacunes et des échecs d'une gestion excessivement centralisée est maintenant généralisée parmi tous les acteurs de la scène sociosanitaire (à la base comme au sommet).

Aussi, dans les termes des documents successifs (du rapport Rochon au document Côté), une plus grande « décentralisation » est une des stratégies principales proposées. Sur ce sujet, le rapport Rochon ainsi que les deux documents gouvernementaux d'orientation tiennent un discours et font des propositions qui vont essentiellement dans le même sens. La " décentralisation » vers les régions est une nécessité pour assurer l'adaptation aux conditions locales, la coordination et l'intégration des services, la responsabilisation des acteurs régionaux et locaux, la motivation des professionnels, I'implication de la population et de toutes les forces sociales à la fois dans le développement social et la gestion des services sociosanitaires.

En continuité avec ce discours, le rapport Rochon et les deux documents gouvernementaux proposent un renforcement sensible du palier régional au moyen de deux stratégies. D'une part, les conseils régionaux seraient transformés en « régies » aux pouvoirs beaucoup plus étendus. Les régies conserveraient les anciens mandats $\mathrm{d}^{\prime}$ " assurer la participation » et de « favoriser la concertation », mais elles obtiendraient en principe l'autorité sur les questions les plus conséquentes pour l'évolution du système sur le plan régional : la planification / programmation régionale en fonction des besoins locaux, l'organisation des plans de services et l'allocation des crédits aux établissements et organismes en fonction de ces plans. Dans les mots de la commission, « la région régira » (Québec, 1988 : 496). 
La deuxième caractéristique des régies proposées est qu'elles seraient gérées par un conseil d'administration formé uniquement de citoyens (à l'exclusion de salariés ou de professionnels du réseau) représentant les divers établissements et organismes sociosanitaires, mais également d'autres acteurs sociaux de la scène sociosanitaire régionale : élus locaux, représentants des groupes socio-économiques, représentants des organismes communautaires. Une telle formule est présentée comme favorable à la fois à la participation active des citoyens et à la démocratisation régionale des débats et des choix. Bref, extension des pouvoirs, autonomie, imputabilité (vers les populations locales et vers le haut), participation, démocratisation sont les objectifs visés.

Du rapport Rochon aux documents ministériels, les propositions de décentralisation ont quelque peu changé. Le rapport Rochon recommandait l'élection au suffrage universel (après une période transitoire) et suggérait qu'à moyen terme les régies aient le pouvoir de taxer. Ces deux recommandations n'ont pas été retenues par les autorités gouvernementales. Pour la constitution des régies régionales, les deux documents ministériels proposent la solution suggérée par le rapport Rochon pour la période transitoire : l'élection du conseil d'administration des régies par un collège électoral (dit aussi assemblée régionale) représentant les divers intérêts et groupes régionaux.

Bref, le rapport Rochon a plaidé pour une décentralisation conséquente comme une des stratégies de choix pour l'amélioration du système sociosanitaire public. Les documents d'orientation gouvernementaux disent retenir cette orientation, avec cependant deux modifications substantielles : pas d'élections au suffrage universel, pas de pouvoir de taxation. Autrement dit, et ce point est confirmé dans la loi de 1991, le gouvernement a reculé devant une décentralisation "poussée ». Toutefois, les pouvoirs des régies seraient élargis, les citoyens sont conviés au débat et à la prise de décision, et, à défaut d'une décentralisation de l'imputabilité politique, des mécanismes précis d'imputabilité régionale et vers le centre sont proposés.

Depuis 1971, la situation s'est-elle modifiée au point que les chances de la décentralisation soient maintenant plus élevées ? Certes, bien des choses ont changé. Deux des raisons invoquées par M. Castonguay au moment du démarrage de la réforme pour expliquer la timidité de la décentralisation ne peuvent être retenues pour ce qui est de la période plus récente. Les « compétences administratives » existent maintenant. Par ailleurs, même si cet indicateur de performance est imparfait, la gouverne étatique centrale a permis une certaine rationalisation du développement du réseau. De plus, le Ministère et les conseils régionaux se sont dotés d'outils efficaces pour un contrôle serré des dépenses (les déficits sont maintenant chose rare).

Mais surtout, les vingt années de travail et d'expérience des CRSSS ont changé la carte sociosanitaire du Québec : les réalités régionales ont pénétré à l'intérieur de la machine administrative et politique; sous le coup de la 
nécessité, les mouvements de concertation / collaboration régionaux se sont intensifiés ; finalement, dans un certain nombre de cas au moins, les Conseils régionaux ont réussi à établir leur crédibilité comme maîtres d'œuvre de la planification régionale. Bref, on dispose d'acquis substantiels.

En contrepartie, l'obstacle décisif qui a empêché jusqu'à présent une plus forte décentralisation de la prise de décision subsiste. II n'y aura pas de nouveau palier politico-administratif régional élu avec pouvoirs de taxation (modèle " poussé » de décentralisation sur un mode multi ou unifonctionnel). Dans ses documents d'orientation, le gouvernement choisit une voie mitoyenne entre le statu quo et une réelle décentralisation : il annonce l'extension des zones de compétence des instances régionales parallèlement à l'accroissement de " l'enracinement politico-social » des conseils d'administration et de leur imputabilité, à travers le mécanisme "représentatif » de l'assemblée régionale. Cette solution permettra-t-elle, dans les faits, une plus grande influence régionale sur les décisions?

\section{PREMIER TEST DE VÉRITÉ : LA LOI DE 1991 (PROJET DE LOI 120)}

Cette section analyse la Loi sur les services de santé et les services sociaux et modifiant diverses dispositions législatives (projet de loi 120 devenu le chapitre 42 des Lois du Québec de 1991) à la lumière des quatre critères de décentralisation pour, dans un premier temps, observer le degré de décentralisation formelle et ensuite déterminer dans quelle mesure la nouvelle loi est, comparativement à l'ancienne, plus ou moins décentralisatrice des pouvoirs ministériels au bénéfice des nouvelles régies régionales.

\section{Personnalité juridique}

Un organisme réellement décentralisé peut naître, disparaître ou muter selon le désir de ses membres. L'article 339 de la loi donne au seul gouvernement le pouvoir d'instituer des régies régionales dans chaque région. Donc, non seulement l'organisme est créé par le ministre, mais c'est le cas aussi de la plupart des établissements sous sa juridiction. Les articles 317 à 333 confèrent au ministre seul ce pouvoir : un établissement public ne peut être constitué qu'en vertu de cette loi (317), seul le ministre peut demander la délivrance ou la modification de lettres patentes à cette fin (318 et 322), il doit agréer les fusions, les conversions (325), les intégrations (331) et les dissolutions (333). C'est aussi lui qui délivre les permis d'exercice des établissements (437).

Les régies régionales ont la personnalité juridique des corporations au sens du Code civil (342), mais elles ne peuvent faire elles-mêmes évoluer 
cette personnalité juridique. Pour ces raisons et à l'égard du critère de la personnalité juridique, les régies régionales apparaissent décentralisées car elles ont une personnalité juridique complète, mais le fait que cette personnalité soit à la fois créée et déterminée par le ministre en limite les attributs d'autonomie. L'ancienne loi est donc peu modifée à cet égard par la loi de 1991 : de plus, cette dernière clarifie et consolide les pouvoirs du ministre sur la création des établissements.

\section{Contrôle des élus par les membres}

Les régies régionales reposent sur les assemblées régionales créées dans chaque région (418) pour élire les membres du conseil d'administration, désigner les substituts, approuver les priorités régionales et le rapport annuel.

Les assemblées régionales sont formées à 40 \% de personnes élues par les établissements publics parmi les membres de leurs conseils d'administration ou désignées par les établissements privés, à $20 \%$ de personnes élues de la même manière par les organismes communautaires, à $20 \%$ de personnes élues par les groupes socio-économiques représentatifs et à $20 \%$ d'élus municipaux (421). Elles sont donc formées exclusivement de personnes de la région et élues par des intérêts régionaux.

Les conseils d'administration sont formés de 23 à 25 personnes dont 20 sont élues par les assemblées régionales et choisies dans les mêmes proportions ; une à trois personnes sont cooptées par les premières, tandis que le directeur général et le président de la commission médicale régionale complètent le conseil (397). Le mode de désignation du conseil libère donc ses membres de tout lien formel avec le ministre ou le Ministère, si ce n'est que le ministre peut déterminer la composition des groupes élisant les membres des assemblées régionales (422). Les membres des assemblées régionales et des conseils d'administration ont des mandats de trois ans, ce qui leur assure une indépendance suffisante pour défendre les intérêts régionaux (339 et 425). Bref, bien que les régies régionales ne soient pas libres de fixer les modes de constitution de leurs organes décisionnels, elles comptent sur des membres qui sont affranchis de liens de dépendance avec l'instance centrale, ce qui satisfait au critère de décentralisation.

Cette décentralisation ne tient pas de la démocratie directe, car ce n'est pas la population qui choisit les dirigeants comme dans le cas des administrations scolaires et municipales, mais plutôt les établissements, les groupes régionaux et les municipalités, et encore le font-ils à travers des collèges électoraux ; I'imputabilité politique régionale des dirigeants des régies apparaît dès lors indirecte et mitigée.

Par rapport à l'article 24 de l'ancienne loi, le nouveau régime augmente le nombre de membres du conseil d'administration (de 15 à 23). Il accroît 
quelque peu l'importance du monde municipal (de $13 \%$ à $17 \%$ ) et il diminue légèrement le poids des groupes socio-économiques (de $20 \%$ à $17 \%$ ). Les représentants des établissements conservent le même poids (33\% vs 34 \%). La proportion de médecins change peu sur le plan quantitatif (un membre) ; mais la création de la commission médicale régionale (qui donne au C.A. son avis sur toute une série de questions relatives à la pratique médicale régionale) et la présence de son président au conseil d'administration correspondent, dans les faits, à une présence accrue des médecins dans la prise de décision. Deuxième changement notable : I'entrée en force des groupes communautaires qui obtiennent $17 \%$ des sièges. À souligner

\section{Composition du conseil d'administration des régies régionales et des anciens conseils régionaux}

\begin{tabular}{|c|c|c|c|c|}
\hline \multirow[b]{2}{*}{ Élus municipaux } & $\begin{array}{c}\text { Régies } \\
\text { Chap. } 42 \text { (1991) } \\
\text { (23 membres) }\end{array}$ & \multicolumn{3}{|c|}{$\begin{array}{c}\text { CRSSS } \\
\text { Chap. } 27 \text { (1981) } \\
\text { (15 membres) }\end{array}$} \\
\hline & $(17,4 \%)^{2}$ & & 2 & $(13,3 \%)^{2}$ \\
\hline Groupes socio-économiques & $4 \quad(17,4 \%)^{2}$ & & 3 & $(20,0 \%)^{2}$ \\
\hline Représentants des établissements & $8 \quad(34,8 \%)^{1}$ & & 5 & $(33,3 \%)^{1}$ \\
\hline Médecins & $1 \quad(4,3 \%)^{1}$ & CMDP & 1 & $(6,6 \%)^{1}$ \\
\hline Organismes communautaires & $(17,4 \%)^{2}$ & Bénév. & 1 & $(6,6 \%)^{2}$ \\
\hline Membre coopté & $(4,3 \%)^{2}$ & & - & - \\
\hline DG régie & $(4,3 \%)^{1}$ & & 1 & $(6,6 \%)^{1}$ \\
\hline Université & - & & 1 & $(6,6 \%)^{2}$ \\
\hline \multirow[t]{2}{*}{ Cégep } & - & & 1 & $(6,6 \%)^{2}$ \\
\hline & $23(99,9 \%)$ & & & $(99,6 \%)$ \\
\hline \multicolumn{5}{|l|}{ Récapitulation } \\
\hline (1) Représentants des producteurs & $10(43 \%)$ & & 7 & $(47 \%)$ \\
\hline (2) Représentants divers des « citoyens » & $13(57 \%)$ & & & $(53 \%)$ \\
\hline \multicolumn{5}{|c|}{$\begin{array}{l}\text { Note : Si la qualification des diverses catégories de membres comme représentants des } \\
\text { "producteurs » ou des " citoyens » est exacte, cela signifierait que malgré le slogan du } \\
\text { " citoyen décideur », la situation post-chapitre } 42 \text { n'aurait guère changé. Cette lecture } \\
\text { doit toutefois rester provisoire jusqu'aux élections. Les représentants des établissements } \\
\text { seront-ils en majorité des cadres du réseau (la loi ne l'exclut pas) ou des " citoyens »? } \\
\text { Il en va de même du « membre coopté ». À noter également que la frontière entre les } \\
\text { deux catégories n'est pas étanche. Même s'ils sont " citoyens », les représentants } \\
\text { des établissements ou des organismes communautaires ne pourront faire entièrement } \\
\text { abstraction des intérêts particuliers des établissement ou des organismes qu'ils repré- } \\
\text { sentent. }\end{array}$} \\
\hline
\end{tabular}


également le fait que le ministre ne nomme plus les représentants des groupes socio-économiques et qu'il n'a pas le pouvoir de nommer des administrateurs si une élection n'a pas lieu dans un collège électoral. En ce sens, le pouvoir indirect du ministre d'influencer les décisions est réduit. C'est le ministre cependant qui nomme le directeur de la santé publique de chaque régie (372). Agissant sur avis de la régie, il a donc un droit de veto sur cette nomination, ce qui est contraire aux principes de la décentralisation. (Pour l'interprétation de ces divers changements, voir le tableau de la page précédente.)

\section{L'exclusivité du champ de compétence}

Un organisme parfaitement décentralisé présente deux caractéristiques quant à son champ de compétence : d'une part, il a des compétences claires, reconnues et complètes au sens où il est seul à décider en ces matières; $d$ 'autre part, aucune autre autorité ne peut envahir son territoire ou son domaine pour exercer de façon concurrente le même champ de compétence. Il va sans dire que cette situation théorique ne trouve pas d'illustrations dans la réalité du monde politico-administratif québécois : les commissions scolaires doivent par exemple composer avec les programmes du ministère de l'Éducation, tandis que les municipalités sont soucieuses de concevoir et de gérer leurs programmes selon les normes du ministère des Affaires municipales qui conditionne ainsi sa contribution financière.

Les objectifs et fonctions des régies régionales sont décrits aux articles 340 et suivants ; la régie doit établir les priorités régionales, mais les priorités retenues doivent tenir compte des objectifs du ministre (340.2) ; en matière de santé et bien-être, la régie informe le ministre des besoins de la population, évalue l'atteinte des objectifs selon la périodicité établie par le ministre, met en œuvre des évaluations selon les directives du ministre et exécute tout mandat confié par le ministre (346).

Les plans d'organisation des services doivent être soumis à l'approbation du ministre (347) après avoir été établis en tenant compte des orientations ministérielles ; la régie est responsable de l'allocation des budgets de fonctionnement dans la mesure et aux conditions prescrites par le ministre (350). Elle gère le programme ministériel de santé publique dans sa région en adaptant les priorités, organisant les services et allouant les ressources (371). Elle élabore, en tenant compte des orientations ministérielles, un plan régional de développement des ressources humaines (376).

Si le ministre émet les permis (437), les demandes de permis lui sont cependant acheminées par la régie (441); de la même façon, le ministre délivre et annule les agréments (457-460), mais la régie donne au ministre son avis sur la question (457). Ce rôle consultatif s'étend au plan régional de répartition des effectifs médicaux (377). 
Les pouvoirs du ministre décrits à l'article 431 sont très étendus, puisqu'il fixe les orientations, priorités, politiques, programmes, cadres de gestion et coordonne interrégionalement les services de santé et sociaux et le programme de santé publique ; c'est encore le ministre qui fixe la politique d'approvisionnement en biens et services (436). La régie doit aussi fournir un rapport annuel au ministre (393) et lui fournir toutes les autres informations demandées (394). En outre, les pouvoirs de réglementation du gouvernement décrits aux articles 505 à 507 et les mandats de négociation des conditions de travail des personnels par le Conseil du trésor risquent de dévorer l'autonomie des régies.

Par contre, les fonctions de coordination des services et de stimulation de la participation de la population (352 et 343) semblent promises à un exercice entièrement autonome. Également, les régies détiennent certaines compétences en propre : I'approbation des paramètres pour les services dispensés par les établissements (105), la réception des plans d'organisation des établissements (183), la gestion du processus de plaintes des usagers (42 et suiv.), la réception des rapports annuels des établissements (278), la coordination des activités des médecins soumis à l'article 360 (352), I'accès des établissements aux ressources intermédiaires (304) et I'arbitrage en cas de conflits (307) et, enfin, la tenue de la séance publique annuelle (384).

Comme gestionnaire des ressources régionales, la régie détient des pouvoirs sur les établissements, pouvoirs qu'elle exerce toutefois le plus souvent dans le cadre des priorités ou orientations ministérielles : elle doit être consultée par les établissements avant la conclusion de contrats en matière de programmes universitaires ou d'ententes internationales (110-111) ; elle peut ou doit donner son avis oral lorsqu'un établissement veut construire, acquérir (260), louer des immeubles (263), convenir de certains contrats (264), acquérir des actions (265) ; elle peut vérifier des informations lorsque des établissements reçoivent des fonds de fondations (279), contrôler certains de leurs emprunts (296), exiger des informations sur leur situation financière (297) ou sur leurs données de gestion (381) ; la régie coordonne régionalement les approvisionnements en commun (383) et applique les plans ministériels de développement des ressources humaines (376) ; sur le plan budgétaire, la régie régionale transmet le budget aux établissements privés conventionnés (299) et verse des allocations financières pour des personnes en perte d'autonomie à des résidences privées ou à des établissements non conventionnés (454).

En somme, les régies jouent pleinement un rôle de coordination, un rôle de fiduciaire de la population ; sur le plan des orientations provinciales, elles ont un rôle d'aviseur du ministre ; en matière de gestion, elles contrôlent régionalement les établissements à l'intérieur des paramètres et directives ministériels. Par rapport à l'article 18 de l'ancienne loi, I'organisme régional a vu s'accroître certaines compétences clés, telles que la définition des 
orientations et priorités régionales et l'allocation intra-régionale des ressources, mais ses pouvoirs à ces égards demeurent sujets à l'approbation ministérielle ; les régies ont plus de pouvoirs qu'avant, mais des pouvoirs qui restent fragiles.

Un tel constat ne peut surprendre dès lors que le contrôle politique des régies n'est ni direct ni démocratique : I'imputabilité politique s'exerce donc à l'Assemblée nationale et la décentralisation cède plutôt le pas à une déconcentration dans laquelle toutefois les agents régionaux jouissent d'un large pouvoir d'influence auprès du Ministère et des établissements ainsi que de pouvoirs importants de gestion et de contrôle sur la scène régionale.

\section{L'autofinancement}

La décentralisation parfaite exigerait une marge certaine d'autofinancement pour que les dépositaires du pouvoir décentralisé puissent être libres d'assumer selon leurs valeurs et préférences les pouvoirs ainsi dévolus. L'organisme décentralisé décide à la fois de ce qu'il fait avec l'argent et de la façon dont il s'y prend pour le dépenser. Qu'en est-t-il dans la nouvelle loi ?

L'article 463 de la loi prévoit que c'est le ministre qui répartit interrégionalement les ressources financières, établit les mécanismes d'allocation et de gestion qui seront utilisés par les régies régionales et approuve les plans d'organisation. Le ministre établit annuellement après approbation par le Conseil du trésor les règles budgétaires d'admissibilité aux subventions (464) et d'allocation des budgets par les régies régionales (465); à cet égard, il se garde toujours une large possibilité d'intervention discrétionnaire (466, al. 3 et 4 par exemple). Les articles 350 et 351 assujettissent la fonction répartitrice de la régie aux règles ministérielles.

Le budget de fonctionnement de la régie régionale est établi par le directeur général selon les paramètres publiés par le ministre avec obligation d'équilibre (387) ; il doit être approuvé par le ministre qui peut en exiger la réforme ou l'assujettir à des conditions (388). Le pouvoir d'emprunt d'une régie régionale est assujetti au veto ministériel (396). Avec l'approbation du Conseil du trésor, le ministre prend des règlements sur tous les aspects de la gestion des achats, des ressources matérielles et approvisionnements tant pour les établissements que pour les régies régionales (485). Le ministre peut permettre que dans certains cas l'approbation de la régie régionale remplace la sienne (486).

Nulle part donc dans la loi n'est prévu l'autofinancement (même partiel) des régies ; les apports financiers extérieurs même minimes sont contrôlés ; les pouvoirs ministériels de contrôle à distance de la gestion semblent même plus importants que ceux prévus aux articles 173 à 180 de l'ancienne loi. 


\section{Bilan}

L'examen sommaire de la loi de 1991 sous l'angle de la décentralisation a montré que les régies ont une personnalité juridique propre bien que celle-ci soit contrôlée par le ministre.

Il a permis de vérifier que les responsables politiques des régies sont libres de liens avec le Ministère ou le ministre et jouissent de mandats de trois ans ; de plus, aucune personne nommée par le ministre ne siégera au conseil d'administration des régies. Toutefois, leur imputabilité s'exerce non pas envers la population régionale, mais envers le collège électoral sectoriel de provenance et l'assemblée régionale.

Les régies n'ont pas réellement de pouvoirs stratégiques sur le plan des compétences : elles doivent appliquer régulièrement les orientations ministérielles et appliquer les règles budgétaires selon les directives du ministre ; leur pouvoir de contrôle sur les établissements s'exerce aux nom et profit du ministre ; les seuls champs de compétence réelle sont la gestion des relations avec la population et la coordination régionale. Dès lors, elles apparaissent comme des bureaux régionaux du Ministère, mais dont les têtes dirigeantes seraient cooptées par le réseau régional. Malgré tout, la nomenclature de leurs champs de compétence est plus vaste que sous l'ancienne loi et leur pouvoir sur les établissements plus grand. Finalement, aucun facteur d'autofinancement n'a été observé.

En somme, les régies sont des organismes formellement décentralisés de par leur personnalité juridique, corporativement décentralisés de par leur direction politique, peu décentralisés de par les compétences exercées et centralisés de par leur financement.

Par rapport à l'ancienne loi, l'évolution vers la décentralisation s'observe quant à l'autonomie dans la sélection des dirigeants régionaux et quant à l'accroissement des compétences intra-régionales des régies, notamment dans leurs rapports avec les établissements. Cependant, le pouvoir d'autofinancement reste absent, tandis que les immenses pouvoirs de réglementation de la gestion que détiennent les organismes centraux (gouvernement, Conseil du trésor, ministre) font peser sur les régies une lourde épée de Damoclès. Certains juristes y voient même une "centralisation accrue » (Marleau, $1991: 15)$.

\section{ENJEUX ET PERSPECTIVES}

Les premières déclarations gouvernementales qui ont suivi le dépôt du rapport Rochon ont présenté le renforcement de la décentralisation comme une des stratégies clés pour améliorer le système sociosanitaire. 
Dans cette perspective, deux voies s'offraient au gouvernement. La première aurait consisté, dans la ligne des recommandations de la Commission, en une réelle décentralisation (élection des dirigeants, pouvoir de taxation), cette option pouvant se concevoir soit sur le mode de la décentralisation unifonctionnelle (de façon analogue aux commissions scolaires), soit par la création d'un palier politico-administratif régional multifonctionnel (analogue aux municipalités ou aux MRC). La deuxième voie comportait le maintien d'organes du type CRSSS en tant qu' « antennes régionales » du Ministère, en étendant leurs zones d'autorité et en clarifiant les rôles respectifs du centre et de l'organisme régional. Avec ses documents d'orientation et la loi de 1991, le gouvernement a en quelque sorte opté pour une formule proche de la deuxième voie : des pouvoirs plus clairs pour l'autorité centrale et la précision des pouvoirs régionaux jointe à l'imputabilité des conseils envers les milieux institutionnels et politiques régionaux. Les régies ne sont pas des organismes entièrement décentralisés (aucun pouvoir de taxation, pas de dirigeants élus au suffrage universel, peu de zones de compétence exclusive), mais leurs dirigeants sont tout de même élus par des groupes régionaux et l'analyse de la loi de 1991 montre que - sur papier au moins - les régies pourraient disposer, sous réserve d'approbation ministérielle, de pouvoirs importants en ce qui concerne les deux décisions stratégiques clés : la définition des orientations et objectifs du système sociosanitaire régional et l'autorité de définir et de contraindre les moyens régionaux (allocation des budgets et ressources) pour l'atteinte des objectifs.

En somme, sans imputabilité politique directe, l'instance régionale ne peut enlever au centre politique et technocratique les responsabilités - de financement, d'orientation, d'évaluation et de contrôle - que les citoyens lui ont confiées. Toutefois, par la participation à la définition des priorités régionales et à l'allocation des ressources, l'organe régional devient un lieu de plus grande transparence du débat politique et administratif. Les optimistes parient que la décentralisation de la négociation (sur les deux enjeux clés : orientations/priorités et allocations des ressources), des relations de pouvoir et de la programmation/gestion des opérations conduira à une prise de décision plus efficace, à des décisions plus adaptées et à un accroissement de la motivation des acteurs régionaux et locaux. Quant au centre, il gagnerait en efficacité opérationnelle (se concentrant enfin sur ses responsabilités stratégiques propres). Bref, le Ministère restera le décideur ultime, mais, pour les optimistes, la décentralisation ne pourra que progresser, car un certain nombre de forces poussent dans cette direction.

En effet, les débats des récentes années ont fait apparaître un consensus généralisé en faveur du renforcement d'un palier régional qui encouragerait la participation des intervenants institutionnels et politiques dans la programmation et la gestion. Au sommet, on s'est finalement rendu compte que 
la centralisation engorgeait un centre ne possédant pas non plus la meilleure connaissance possible des réalités régionales (Québec, 1990). De plus, le gouvernement ayant suffisamment claironné ses intentions de décentraliser, la pression politique de livrer la marchandise sera présente. Les optimistes parient aussi que, peu importe les pouvoirs formels, le pouvoir réel des régies ira en s'accroissant, car elles détiennent et détiendront de plus en plus un élément clé du pouvoir, l'information sur les réalités régionales. Au vu du renforcement progressif des conseils durant la dernière décennie et de la différenciation observée dans le degré de dynamisme des conseils, cette prétention n'est pas sans fondement.

Le progrès réel de la décentralisation reste toutefois incertain. Un consensus généralisé existe, dans un système fondé sur l'équité, à la fois sur la nécessité d'un contrôle administratif et politique de la part du centre comme sur la nécessité de normes provinciales. Dans ce contexte, l'enjeu devient la marge de manœuvre réelle dont les régies bénéficieront en matière d'adaptation régionale des priorités, de gestion des programmes et d'allocation des ressources, d'élaboration de normes et de contrôle des résultats. Les pessimistes redoutent que les normes ministérielles d'agrément des programmes et d'allocation des ressources entament sérieusement le pouvoir de décision réel des régies.

Un premier test, le libellé de la loi de 1991, a été discuté. La loi accorde bien plusieurs des pouvoirs promis dans le document Côté, mais il est frappant de constater que non seulement la régie doit faire approuver par le ministre l'exercice de ses fonctions principales, mais aussi que le ministre se réserve toute une série de fonctions et de contrôles qui pourraient limiter substantiellement l'étendue réelle de la décentralisation. Il est classique pour les ministres de se réserver, à titre préventif, d'amples pouvoirs qu'ils n'utiliseront pas ou avec discernement, mais ici l'épée de Damoclès centralisatrice et bureaucratique est loin d'être absente. En définitive, que prévoira la réglementation quant aux pouvoirs administratifs des régies ? On pourra mesurer si le gouvernement passe de la parole aux actes.

Par ailleurs, le renforcement de l'instance régionale, elle-même liée à I'instance centrale, correspond à première vue à une " centralisation régionale ", contre laquelle s'insurgent ceux qui défendent les corporatismes locaux, la sacro-sainte autonomie des établissements. Cette centralisation peut toutefois engendrer une bureaucratisation régionale excessive. À court terme, vu la pénurie de ressources, le danger d'absence $d^{\prime}$ une bureaucratie suffisante est possiblement plus grand que celui d'une bureaucratisation régionale excessive. Toutefois, I'ampleur des tâches que le ministre se réserve de demander au personnel des régies fait craindre que les régies (ainsi que les établissements par ricochet) soient prises dans un dédale bureaucratique. Ce risque pourrait cependant diminuer grâce à la mise sur pied de modes de gestion axés sur les résultats obtenus plutôt que sur les moyens investis. 
Un deuxième test devrait avoir lieu bientôt. Plusieurs pensent que les régies ne pourront remplir les mandats que la loi leur confie sans une augmentation de leur personnel qui, normalement, devrait s'accompagner d'une baisse progressive des effectifs du centre. Verra-t-on ce transfert? Le ministre a promis de l'argent neuf pour les régies, mais ces crédits seront-ils en définitive débloqués malgré la situation actuelle des finances publiques?

À court terme, le pouvoir du Ministère de contraindre les régies à tenir compte de certaines considérations particulières dans l'allocation des budgets aux établissements régionaux peut, en consacrant des « rentes de situation », entamer à la fois le pouvoir des régies d'allouer rationnellement les ressources et leur crédibilité.

Le gouvernement réussira-t-il par ailleurs à endiguer les courtcircuitages administratifs et politiques qui ont tellement miné les conseils régionaux ? La réponse à cette question dépendra, notamment, de la cure d'amaigrissement que devrait subir le MSSS tant dans ses effectifs que dans ses prérogatives et processus ; elle viendra aussi du développement réel des régies dans les prochaines années (problème de ressources) et de la dynamique décisionnelle qui s'installera dans les régions (leadership des régies, degré de réussite de la concertation, etc.). La cooptation des médecins, la négociation des programmes et plans de services par les divers acteurs régionaux (sans doute surveillée politiquement par le centre comme sur le plan local) constituent ici des éléments favorables.

Autre incertitude : dans quelle mesure les nouveaux conseils d'administration pourront-ils mieux que par le passé agir en fonction des intérêts des citoyens plutôt qu'en fonction des pressions des producteurs ? Le gouvernement $n^{\prime}$ aura pas les mêmes responsabilités $d^{\prime}$ influence directe puisque les nominations gouvernementales ont disparu. Toutefois, malgré le slogan du " citoyen décideur », on sera surpris de constater que, par rapport aux anciens conseils, il n'y a pas de changement quantitatif notable de la proportion de membres du conseil d'administration ayant des liens directs avec les producteurs ( $43 \%$ pour les régies comparativement à $47 \%$ pour les CRSSS ; voir tableau page 103). En outre, en termes absolus, les représentants des établissements seront plus nombreux dans le nouveau conseil (huit $v s$ cinq) et pourraient comprendre une portion importante de cadres ou de professionnels du réseau ${ }^{7}$. On se demande dans quelle mesure les « gens ordinaires » pourront manipuler les pièges du langage et des enjeux technocratiques.

De plus, les producteurs disposent de moyens supplémentaires pour influencer les décisions (lobbying, noyautage des conseils, présence dans les comités consultatifs). Bref, certains craignent que les intérêts des producteurs pourraient rester dominants. La situation est toutefois ouverte : I'entrée en force des organismes communautaires pourrait signifier une plus grande 
sensibilité aux besoins réels de la région. Également, les producteurs ne constituent pas un groupe homogène : hôpitaux, CLSC et centres d'accueil ne font pas nécessairement bloc. En tout état de cause, les représentants des producteurs devront faire face à un éventail de représentants de divers groupes de la communauté. En outre, le fait que les séances des conseils d'administration (régies et établissements) seront dorénavant publiques pourrait constituer un « aiguillon » important sur le plan de l'imputabilité aux besoins des citoyens ${ }^{8}$.

Dans le même ordre d'idée : le conseil d'administration et l'assemblée régionale pourront-ils contrebalancer le pouvoir de la « technostructure régionale » ? À quelles conditions ? L'expérience nord-américaine avec les conseils d'administration du secteur de la santé montre qu'au moins trois conditions doivent être remplies pour un fonctionnement fécond d'un conseil d'administration : une formation préalable des membres, la distribution à temps d'une information appropriée et la nature de l'agenda du conseil : les citoyens sont intéressés par des enjeux réels (tels les débats sur les priorités ou les services offerts) et non par des détails administratifs. Par ailleurs, plusieurs observateurs pensent qu'une volonté et une capacité de participation sont en train d'émerger sur le plan local ; les citoyens pourraient poser les bonnes questions à la fois sur les conditions liées à la santé et sur la nature des services étatiques ou communautaires. À certaines conditions de transparence des débats et de fréquence des réunions, l'assemblée régionale pourrait aussi constituer un lieu important d'imputabilité envers les besoins régionaux.

Au bilan, les acteurs régionaux ont gagné plus de latitude pour adapter la programmation centrale et déterminer le mode de distribution des services sociosanitaires régionaux. Il ne s'agit pas d'une décentralisation authentique avec pouvoir de taxation, élection au suffrage universel et zone de compétence exclusive, mais la loi rend possible un renforcement de I'influence régionale sur certaines décisions stratégiques. On pourrait presque qualifier les régies de "bureaux régionaux politico-corporatistes », car le pouvoir du centre reste, sur papier, très fort et menaçant... Toutefois, le gouvernement propose une nouvelle forme de gestion " politico-corporatiste » des questions sociosanitaires régionales fondée sur la concertation régionale faisant appel à la fois aux forces politiques et sociales régionales et aux intervenants corporatistes. Cette formule «intégrative » pourrait conduire à une bonification du secteur sociosanitaire.

Le gouvernement pouvait-il aller plus loin ? II faut d'abord souligner que le secteur sociosanitaire mobilise des ressources publiques immenses et qu'un certain nombre des enjeux qu'il soulève (le financement, la distribution équitable des services sur l'ensemble du territoire, la prévention, les rapports interprofessionnels, etc.) sont d'une telle importance qu'il est impensable que le centre politique abandonne la définition des orientations, des programmes de base, des normes générales de services et de financement. On ne peut 
décentraliser le secteur sociosanitaire au même titre que l'entretien des trottoirs ou le choix local des loisirs. En ce sens, les jérémiades contre la bureaucratie centrale et les appels scandalisés à une vraie décentralisation politique ne tiennent pas compte de tous les éléments du problème.

Ces appels surestiment aussi la possibilité de créer au Québec, à court terme au moins, de nouvelles structures politico-administratives nécessaires à une réelle décentralisation politique. D'une part, est-il réaliste de songer à la création d'un palier politico-administratif régional multifonctionnel dans le contexte de la remise en cause des coûts des services étatiques ? D'autre part, la réflexion sur les instances supramunicipales se poursuit. Même si l'on parle de plus en plus au Québec des "réalités régionales », le dernier document gouvernemental sur la politique régionale ne fait pas allusion à la création d'un vrai palier politico-administratif régional (Québec, 1991b).

L'idée d'un palier politico-administratif unifonctionnel, dans la ligne des recommandations de la Commission Rochon, n'apparaît pas plus mûre. À cet égard, il faut bien constater que la Commission n'a pas été très loquace. Elle proposait un transfert important des pouvoirs, entre autres du " pouvoir de dépenser », et proposait que les régies puissent à moyen terme lever des impôts. Mais les questions cruciales ont été laissées en plan : quelle part du financement serait régionale ? Si cette part devait être marginale (comme le laissa entendre le président en conférence de presse), quel serait le changement ? Si la majeure partie du financement vient du centre ou est centralement normée, comme pour les commissions scolaires, que deviennent l'autonomie régionale et la participation des citoyens vu l'inévitable imputabilité politique des élus provinciaux ? Inversement, si le financement est sensiblement décentralisé, comment concilier des normes provinciales de service avec la liberté d'action des élus régionaux ? Sans réponse appropriée à ces questions, la portée de la proposition est difficilement appréciable.

En conclusion, avec la loi de 1991, on peut parler de « changement dans la continuité »... même si l'évolution semble à certains très lente et trop timide... L'obstacle majeur qui a handicapé l'autonomie des CRSSS - I'absence d'un statut politique régional au sens classique - n'est pas levé. Toutefois, l'extension de la " compétence partagée » des régies, le nouveau système d'imputabilité aux acteurs régionaux dont l'impact reste à évaluer, la croissance du pouvoir réel (par opposition à formel) des régies pourraient conduire à un renforcement ultérieur de l'instance régionale. Bref, la loi de 1991 autorise des espoirs chez certains qui croient observer déjà certains transferts de pouvoirs et de personnels, et qui misent sur l'intense volonté politique du ministre ; d'autres croient plutôt qu'encore une fois la bureaucratie centrale résistera à la dévolution, justifiant la survie de ses prérogatives actuelles par la crainte du dérapage financier consécutif au changement des acteurs administratifs et à la pression de la demande régionale. 


\section{Notes}

1. Par " essentiellement public », il faut entendre d'une part que le financement est largement socialisé (79\% des dépenses de santé sont financées publiquement) et, d'autre part, qu'une partie prépondérante des services est fournie par des organisations publiques.

Par ailleurs, la gouverne publique du sytème sociosanitaire a permis des progrès notables sur plusieurs plans : accessibilité, progrès dans l'état de santé (ex. : périnatalité), etc.

2. Plusieurs acteurs (associations d'établissements, médecins, organismes communautaires même) ont profité des débats récents pour exprimer leurs sempiternelles récriminations contre la bureaucratie gouvernementale, mais rares sont ceux qui ont clairement préconisé une remise en question sérieuse du rôle public dans le secteur sociosanitaire.

3. Lemieux (1990) propose essentiellement la même distinction en trois critères, tenant pour acquise la personnalité juridique.

4. À noter également que la Commission Castonguay-Nepveu préconisait une plus forte participation de la population dans les orientations et la gestion du secteur sociosanitaire que celle qui s'est manifestée par la suite. Sur les plans régional comme local, la Commission préconisait une double structure de participation : une " participation décisionnelle » aux conseils d'administration, une " participation consultative » à travers la création de conseils consultatifs composés uniquement (au niveau local) et très majoritairement (au niveau régional) de citoyens représentant divers groupes et intérêts.

5. La participation de la population subit la même cure d'amaigrissement : les conseils consultatifs disparaissent et les représentants de la population générale aux conseils régionaux seront réduits à la portion congrue.

6. Ce n'est pas le lieu ici de traiter en détail la nature du développement régional et local, sur ses divers plans, au Québec. Il s'agit d'un phénomène en plein mouvement, diversifié, aux multiples dimensions et dont les tenants et aboutissants sont loin d'être définis. Il y a des régions en déclin (Conseil des affaires sociales, 1989) et des régions en développement. La relation entre le développement régional et le développement local constitue également un phénomène mouvant. Quant aux politiques gouvernementales, des MRC aux instances régionales, elles sont, depuis une dizaine d'années, en évolution constante.

7. Un glissement dont l'impact est a priori difficile à évaluer s'est produit entre les documents d'orientation et la loi. Dans les premiers, les employés et professionnels du réseau étaient exclus du conseil d'administration de la régie, ce qui a donné lieu à l'émergence du slogan du « citoyen décideur ». Ce n'est plus le cas dans la loi de 1991 qui permet aux représentants des établissements (contrairement à ceux des organismes communautaires) de comprendre des employés ou professionnels du réseau.

8. Il faudra attendre la promulgation des règlements et les élections pour évaluer l'impact du processus d'élection en deux étapes (assemblée et régie). Les souscollèges électoraux éliront-ils leurs représentants en vase clos, ou bien les candidats devront-ils gagner le soutien d'autres groupes, ce qui pourrait conduire à l'élection de représentants d'établissements choisis en fonction de leur compétence et de leur capacité de s'élever au-dessus des intérêts particuliers? 


\section{Références bibliographiques}

AuCOIN, Peter et Herman BAKVIS (1988). The Centralization-Decentralization Conundrum : Organization and Management in the Canadian Government. Institut de recherche sur les politiques publiques, Halifax.

BÉLANGER, Jean-Pierre (1990). "Local Community Service Centers : a Contribution to Promotive Services in Quebec / Canada », unpublished manuscript, November 1990.

BHÉRER, Harold et al. (1986). Le miroir magique de la décentralisation. Rapport de recherche. Québec : Gouvernement du Québec, ministère de la Santé et des Services sociaux.

CONSEIL DES AFFAIRES SOCIALES (1989). Deux Québec dans un, Rapport sur le développement social et démographique. Boucherville : Gaëtan Morin éd.

DuSSAULT, René et Louis BORGEAT (1984). Traité de droit administratif, Tome I, $2^{\mathrm{e}}$ édition. Québec : Presses de l'Université Laval.

FIELD, Mark (1989). Success and Crisis in National Health System : a Comparative Approach. New York : Routledge.

GÉLINAS, A. (1975). Organismes autonomes et centraux. Sillery : Presses de l'Université du Québec.

LEMIEUX, Vincent (1990). "Présentation. La Santé. Le système et les acteurs », Recherches sociologiques, vol. 31, $\mathrm{n}^{\circ} 3: 329-337$.

MARLEAU, Véronique (1991). «La réforme du système québécois de la santé - Premier bilan », Bulletin de la Corporation professionnelle des médecins du Québec, vol. 31, no 4, novembre 1991.

QUÉBEC (1970). Rapport de la Commission d'enquête sur les services de santé et le bien-être social, vol. 4, La Santé. Québec : Gouvernement du Québec.

QUÉBEC (1988). Rapport de la Commission d'enquête sur les services de santé et les services sociaux. Québec : Les Publications du Québec

QUÉBEC (1989). Pour améliorer la santé et le bien-être au Québec : Orientations. Québec : Gouvernement du Québec, ministère de la Santé et des Services sociaux.

QUÉBEC (1990). Une réforme axée sur le citoyen. Québec : Gouvernement du Québec, ministère de la Santé et des Services sociaux.

QUÉBEC (1991a). Loi sur les services de santé et les services sociaux et modifiant diverses dispositions législatives (projet de loi 120 devenu le chapitre 42 des Lois du Québec de 1991). Québec : Éditeur officiel du Québec.

QUÉBEC (1991b). Rapport-synthèse, Groupe de travail interministériel sur le développement régional, octobre 1991.

SAltMAN, Richard et Casten von OtTER (1992). Planned Markets and Public Competition (Strategic Reforms in Northern European Health Systems). Philadelphia : Open University Press.

TURGEON, Jean (1983). Évaluation du processus de décentralisation administrative vers le niveau régional en affaires sociales. Québec : Gouvernement du Québec, ministère des Affaires sociales.

TURGEON, Jean (1987). CRSSS et décentralisation de 1972 à 1987 ; Bilan d'une recherche. Québec : Gouvernement du Québec, ministère de la Santé et des Services sociaux. 